\title{
Altered Spontaneous Brain Activity Patterns in Patients with Age-related Macular Degeneration: A Resting-State fMRI Study Running head: Functional Connectivity Density alterations in age-related macular degeneration patients
}

\section{Rong Huang}

The First Affiliated Hospital of Nanchang University

\section{Li-Juan Zhang}

The First Affiliated Hospital of Nanchang University

Min-Jie Chen

The First Affiliated Hospital of Nanchang University

Qian-Min Ge

The First Affiliated Hospital of Nanchang University

Hui-Ye Shu

The First Affiliated Hospital of Nanchang University

Yi-Cong Pan

The First Affiliated Hospital of Nanchang University

Rong-Bin Liang

The First Affiliated Hospital of Nanchang University

Qiu-Yu Li

The First Affiliated Hospital of Nanchang University

Yi Shao ( $\sim$ freebee99@163.com)

The First Affiliated Hospital of Nanchang University

\section{Research Article}

Keywords: age-related macular degeneration, degree centrality, spontaneous brain activity, resting state, MRI

Posted Date: August 10th, 2021

DOI: https://doi.org/10.21203/rs.3.rs-753970/v1 
License: (c) (i) This work is licensed under a Creative Commons Attribution 4.0 International License. Read Full License 


\section{Abstract \\ Background}

Many neuroimaging studies have confirmed that age-related macular degeneration (AMD) is closely related to the changes of brain functional areas. However, the value of spontaneous brain activity changes induced by AMD in early diagnosis of diseases has not been confirmed.

\section{Purpose}

To explore potential functional network brain activity in patients with AMD using the voxel-wise degree centrality (DC) method and reveal the functional changes of specific brain regions in patients with AMD.

\section{Material and Methods}

Eighteen patients with AMD (10 males, 8 females) and eighteen healthy controls (HCs) (10 males, 8 females) matched by gender, age, and education level were enrolled. The resting-state fMRI scanning was applied to measure changes in initiative brain activities. We displayed the differences between the AMD group and the healthy group with the receiver operating characteristic curves. Using correlation analysis, the average DC values in specific cerebrum regions were analyzed to explore changes of behavioral performance in AMD patients. The Hamilton Rating Scale for Depression and Anxiety (Ham-D/A) was used to evaluate the relationship between emotional states and DC values of the subcallosal gyrus in AMD patients.

\section{Results}

In AMD patients, DC values were significantly lower in the bilateral lingual gyrus but were higher in the bilateral inferior temporal gyrus, bilateral subcallosal gyrus, bilateral fusiform gyrus, bilateral middle frontal gyrus, and left orbital middle frontal gyrus/insular gyrus/inferior frontal gyrus. There was no direct relationship between the behavioral performance and the average DC values of some areas.

\section{Conclusion}

The DC values changed with the fluctuation of spontaneous brain activities in AMD patients, which may be beneficial for clinical diagnosis.

\section{Introduction}


Age-related macular degeneration (AMD) is a medical problem characterized by acquired and progressive deterioration, the most typical pathological feature of which is vision loss[1]. Loss of central vision caused by retinal atrophy or neovascularization is the result of abnormal cells (retinal epithelial and pigment cells) and membrane structures (choroid and sclera) [2] . There are two major types of AMD, wet (exudative) and dry (nonexudative). Dry AMD is characterized by the distribution of yellow-white glass membrane warts with limitations in the macular area and near-circle, appearing as atrophic lesions located in the macular area and nearby retina and choroid [3]. Wet AMD may be accompanied by intraretinal hemorrhage and exudation, characterized by choroidal neovascularization and macular discoid scar [4]. The most common pathological type is dry AMD. Wet AMD, although not as common as dry AMD, can progress faster to dvanced hypopsia [3]. A study showed that macular degeneration is hereditary, and some specific genetic loci are highly correlated with macular degeneration, leading to a significant higher risk of AMD in future generations compared with the normal population [5]. In developed countries, many elderly people become blind because of macular degeneration, and this loss of vision is irreversible. Some people predict that by 2040 , the number of patients with AMD worldwide will reach 288 million, and the morbidity rate of AMD in early, late and any other stages will be $8.01 \%$, $0.37 \%$, and $8.69 \%$, respectively $[6,7]$. Previous study have found that $28 \%$ of AMD patients may progress from middle stage to late stage within five years[8]. Anti-vascular endothelial growth factor drugs are a highly recognized treatment scheme at present, but they need to be used for a long time, which affects patients' compliance[9]. Moreover, the treatment effect of this method is not good in the middle and late stage of AMD[8]. Therefore, the prevention and early diagnosis of AMD are very important. Relevant preventive measures include quitting smoking and controlling hypertension[10]. And for the early diagnosis of AMD, functional magnetic resonance imaging (fMRI) technology has been proved to have a good application prospect[11].

Recently, magnetic resonance imaging (MRI) technology has demonstrated its pivotal role in the research of various neuropsychiatric diseases. MRI can be used to detect temporal and spatial correlations of low frequency activity fluctuations in various brain areas, which can be used to evaluate spontaneous brain activities [12]. The DC method is included in resting-state fMRI, which monitors network structures and functional connections of various cerebral regions at the voxel level [13]. It is the simplest and most effective measure to describe the central node evaluation of network connections; the degree of the node reflects its centrality and importance. In the exploration of the neural mechanism of many other diseases [14-19] , the DC method has frequently been adopted to identify altered spontaneous brain activity, with good evaluation results. However, the application value of DC method in the diagnosis of brain function changes in AMD has not been confirmed. In the present study, the underlying relationships between spontaneous changes in brain activities and clinical characteristics of patients with AMD was further explored using the DC method.

\section{Subjects And Methods}

\section{Subjects}


We enrolled 18 patients with AMD (10 men and 8 women) and $18 \mathrm{HCs}$ from the First Affiliated Hospital of Nanchang University and they were highly matched by gender, age, weight, handedness and education level. All subjects included in this experiment met the following criteria: 1) at least 50 years of age; 2) absence of brain parenchyma damage; 3) absence of neuropsychiatric disorders; 4) secondary or primary choroidal neovascularization in the macular area; and 5) fundoscopy and fluorescein fundus angiography revealing pathological characteristics of AMD (Figure 1); 6) no history of diabetes. The study met the ethical standards of the Medical Ethics Committee of the First Affiliated Hospital of Nanchang University as well as the ethical standards of Declaration of Helsinki. We explained the materials, methods ,purpose, and underlying risks of this experiment in advance, and obtained informed consent from all participants.

\section{MRI parameters}

Using a Trio 3-Tesla MR scanner (Trio; Siemens, Munich, Berlin, Germany), under the same physical conditions, we completed MRI scanning for each participant. All patients remained awake during the scanning, with eyes closed and breathing normally until the experiment was completed [20].The spoiled gradient-recalled echo sequence was applied to obtain whole-brain T1-weights. Revelant parameters were as follows: echo time $=2.26 \mathrm{~ms}$, repetition time $=1900 \mathrm{~ms}$, field of view $=250 \times 250 \mathrm{~mm}^{2}$, acquisition matrix $=256 \times 256$, thickness $=1.0 \mathrm{~mm}$, flip angle $=9^{\circ}$, and gap $=0.5 \mathrm{~mm}$. We performed $\mathrm{fMRI}$ with these relevant parameters: echo time $=30 \mathrm{~ms}$, repetition time $=2000 \mathrm{~ms}$, field of view $=220 \times 220 \mathrm{~mm}^{2}$, acquisition matrix $=64 \times 64$, thickness $=4.0 \mathrm{~mm}$, flip angle $=90^{\circ}$, gap $=1.2 \mathrm{~mm}$, and 29 axials.

\section{fMRI data processing}

We pre-filtered datas using MRIcro (www.MRIcro.com) to avoid the interference of invalid datas. We preprocessed the filtered data using SPM8 (https://www.fil.ion.ucl.ac.uk/spm/), the Resting-state Data Analysis Toolkit (http://www.restfmri.net/forum/), and DPARSFA (http://rfmri.org/DPARSF). To obtain a balance among signals and to make the participants adapt to the noise generated during scanning, under normal circumstances, the first few volumes of functional magnetic resonance images were regarded as invalid data and were excluded. Second, the remaining functional BOLD images are checked for slice head motion, and we filtered out subjects with excessive head movement. The standard is no more than 2 $\mathrm{mm}$ in the $\mathrm{X}, \mathrm{Y}$, or $\mathrm{Z}$ directions of the maximum offset. Then, a Gaussian filter was used for smoothing pretreatment to remove the linear tread, and finally we used a band-pass filter to reduce the influence of physiological noise on experimental results. This method has been described in detail [21-23].

\section{Degree centrality}

To calculate DC values, we calculated the total correlations that significantly exceeded the threshold of every participant on the basis of a single-voxel functional network. Then, to transform every individual voxel-wise $D C$ diagram into a $z$-score map, we adopted the following conversion formula [24]: $Z_{i}=D C_{i}$ mean $_{\text {all }} /$ std $_{\text {all }}\left(Z_{i}\right.$ refers to the voxel $z$-score and $D C_{i}$ refers to its voxel-wise $D C$ value, mean ${ }_{a l l}$ and 
std $_{\text {all }}$ refer to average DC value and standard deviation of total voxels in our cerebral areas, respectively) [25].

\section{Correlation analysis}

We applied correlation linear analysis to analyze the associations between the average DC value of various cerebral areas and clinical characteristics, and then the interesting regions and non-interesting regions were divided. The statistical threshold was $\mathrm{P}<0.05$.

\section{Statistical analysis}

Using SPSS software version 20.0 (IBM Corp, Armonk, NY, USA), we compared the common clinical variables and demographic datas of AMD and healthy controls(HCs) using independent sample t-tests at the significance level of $5 \%$. Using receiver operating characteristic (ROC) curves, the mean DC values in various cerebral regions of $\mathrm{HCs}$ and patients with AMD were classified. To determine whether the average DC value of each brain region is related to the clinical behavior of AMD patients such as anxiety or depression, we used the Pearson correlation analysis to describe the relationship between the two groups.

\section{Results}

\section{Demographics and behavioral results}

The 18 patients with AMD (10 males and 8 females) and the $18 \mathrm{HCs}$ ( 10 males and 8 females) showed no significant differences in terms of gender $(P>0.99)$, weight $(P=0.542)$ \age $(P=0.794)$, IOP-left $(P=$ 0.824), or IOP-right $(P=0.786)$. However, statistically significant differences were discovered in the bestcorrected VA-left $(P=0.007)$ and the best-corrected VA-right $(P=0.004)$. (Table 1$)$

\section{Differences in DC}

In comparison with $\mathrm{HCs}$, patients with $\mathrm{AMD}$ represented greatly reduced $\mathrm{DC}$ values in the bilateral lingual gyrus, but increased DC values in the bilateral fusiform gyrus, bilateral subcallosal gyrus, bilateral inferior temporal gyrus, bilateral middle frontal gyrus, and left orbital middle frontal gyrus/insular gyrus/inferior frontal gyrus (Figures 2aख2b and Table 2). Figure2c shows the average DC values between the patients with AMD and HCs.

\section{Receiver operating characteristic curves}

Based on our experimental results, we suspected that differential progress of AMD might be reflected by altered DC values, which will help measure the progression of AMD in clinical use. ROC curves was generated to show the average DC values in specific brain regions. The following datas are the values for the areas under the ROC curves (AUCs) in various brain areas: bilateral fusiform gyrus $(0.889)$, bilateral inferior temporal gyrus (0.940), bilateral subcallosal gyrus (0.861), bilateral middle frontal gyrus $(0.858)$, 
and left orbital middle frontal gyrus/insular gyrus/inferior frontal gyrus (0.920) (AMD >HCs; Figure 3a); bilateral lingual gyrus (0.957) (AMD < HCs; Figure $3 b)$.

\section{Correlation analysis}

For patients with AMD, anxiety scores positively related with DC values of the subcallosal gyrus $(r=0.947$, $p<0.001)$. Depression scores also had a positive relationship with $D C$ values in the subcallosal gyrus $(r=$ $0.826, p<0.001)$. $\otimes$ Figure $4 \square$

\section{Discussion}

To the best of our knowledge, we are the first to evaluate functional network brain-activity changes associated with AMD using the DC method (Figure 5). This method has been used to study optic neuritis [26], congenital comitant strabismus [27], high myopia [28] and other diseases, and has made good progress, toward the prospect of clinical application.(Figure 6).

The lingual gyrus is located in the visual joint cortex of the occipital lobe, where it is mainly responsible for vision processing [29]. Studies have shown that glutamate andy-aminobutyrate (GABA) in the occipital cortex are involved in the process of vision development, and local brain function activity of our visual system represented by fMRI decreased with increases in GABA level [30]. Another study used resting-state cortical entropy to express the activity of cerebral cortex after hypopsia, and found that the loss of central vision was associated with increased resting-state cortical entropy [31]. Studies have found that the activity of the lingual gyrus increases with the development of vision [32, 33]. In the present study, DC values of the lingual gyrus in patients with AMD decreased to a certain level in comparison with that of healthy people, suggesting that the activity of the lingual gyrus decreased; nevertheless, it retained a high DC value, which may suggest that the lingual gyrus is very active in brain visual processing. When AMD patients have poor vision, it may maintain a high level of activity to slow the development of vision loss.

The fusiform gyrus lies in the mid-bottom of the visual joint cortex, responsible for face recognition and object secondary classification recognition, which can produce face-like recognition of visual images that are unreal faces [34]. Previously, researchers studied the relationships between the fusiform gyrus and autism [35], epilepsy [36], chronic schizophrenia [37], and found that blood flow in the fusiform gyrus increased with visual stimulation, and that the fusiform gyrus is closely related to vision [38]. The DC value of the fusiform gyrus increased in our study, possibly as a result of compensatory increase of facial classification and recognition in patients with AMD.

The inferior temporal gyrus is part of the temporal lobe that is mainly responsible for speech processing, memory processing, visual perception and integration functions [39]. Grotheer et al. found that the inferior temporal gyrus is selective to visual stimuli, suggesting a function involved in information processing [40]. Previous studies showed that patients without language disorders and language advantages showed declines in language and visual memory after partial resection of the inferior 
temporal gyrus [41-44]. Similarly, we recorded low DC values in the inferior temporal gyrus in patients with AMD, although with an increasing trend compared with the HCs. We speculate that the visual processing ability of the inferior temporal gyrus in patients with AMD has a compensatory effect on vision loss.

The subcallosal gyrus is part of limbic system and is mainly responsible for the regulation of emotion [45]. Subcallosal gyrus dysfunction is related to depression [45], schizophrenia [46], Alzheimer's disease [47], and others. In our study, we adopted the Ham-D/A to evaluate potential emotional changes of patients. We found that scores of depression and anxiety both positively correlated with the DC values of the subcallosal gyrus in patients with AMD; therefore we suspect that loss of vision caused by the disease triggers mood swings, leading to the occurrence of mental diseases.

The middle frontal gyrus is located between the suprafrontal sulcus and the subfrontal sulcus, and is related to memory processing and attention control [48]. The frontal orbital area is located around the intersection of the papillary muscle of the optic nerve and the precentral gyrus, while the precentral gyrus is closely related to eye movement and visual attention [49]. Reseachers reported that, under normal conditions, the middle frontal gyrus can change attention control from exogenous to endogenous [50]. Gohel et al. reported that the functional activity of the middle frontal gyrus increased in patients with glioma, suggesting that the resting-state $\mathrm{fMRI}$ of the middle frontal gyrus can be added to the non-invasive mode list, and can be used in the context of glioma to assess the hemispheric advantage of language and to guide the selection of treatment options [51]. Similarly, a study indicated that the increase in the DC value may be represent a compensatory activation of visual connection in the middle frontal gyrus.

The insular gyrus is anatomically closely connected with the frontal lobe, the parietal lobe and the temporal lobe [52], and is mainly responsible for dealing with sensory experiences produced by convergence information[53]. The insula includes the anterior insular gyrus and the posterior insular gyrus [54]. A previous study found that the insular promoted emotional changes and formation of subjective feelings by integrating sensory information of internal and external sensations; therefore, it is considered to be the center of the significant network [55]. In our study, compared with HCs, the insular lobes showed increased DC values, and this may be a functional compensation for the reduction of integrated internal and external senses, thereby avoiding the separation of senses (Table 3 ).

Our study has some limitations. First, we did not include sufficient samples, which may give rise to unintended errors. Second, the visual impairment caused by AMD may have influence on patient psychology. Before the study, the neuropsychological status of the patients was not evaluated; emotional states might have an impact on the experimental results. Third, the influences of sound and light and other physical factors were not ruled out during MRI examinations. In future studies, we will expand the sample size, assess psychological changes of patients with AMD in advance, and control the influence of physical factors, so as to further study the neuropathological mechanism of patients with AMD.

\section{Conclusion}


In conclusion,patients with AMD show different DC values in various brain regions, suggesting changes in spontaneous brain activities. Changes in DC values suggest the possible physiological and pathological changes of brain areas. It may be beneficial to further explore the pathogenesis and neural mechanism of AMD so that these can be used for clinical diagnosis.

\section{Declarations}

Consent for publication】All authors agree to publish the manuscript.

Availability of data and material: The datasets used and analysed during the current study are available from the corresponding author on reasonable request.

Competing interests: This was not an industry supported study. The authors report no conflicts of interest in this work.

Funding: Key Research Foundation of Jiangxi Province (No: 20181BBG70004); Excellent Talents Development Project of jiangxi Province区20192BCBL23020囚; Natural Science Foundation of jiangxi Province区20181BAB205034『; Grassroots Health Appropriate Technology “Spark Promotion Plan" Project of Jiangxi Province(No:20188003); Health Development Planning Commission Science Foundation of Jiangxi Province (No: 20201032); Health Development Planning Commission Science TCM Foundation of Jiangxi Province (No: 2018A060)

Author contributions: Each author has made a contribution. Rong Huang is responsible for the writing of the manuscript, Li-Juan Zhang are responsible for the formulation and evaluation of the experimental scheme,Min-Jie Chen and Qian-Min Ge is responsible for recruiting and screening experimental subjects, Hui-Ye Shu and Yi-Cong Pan are responsible for preparing experimental materials and executing the experiment, Rong-Bin Liang and Qiu-Yu Li are responsible for data analysis and collation. Shao Yi is responsible for the revision of the paper.

Ethical Statement: All research methods were approved by the committee of the medical ethics of the First Affiliated Hospital of Nanchang University and were in accordance with the 1964 Helsinki declaration and its later amendments or comparable ethical standards. All subjects were explained the purpose, method, potential risks and signed an informed consent form.

\section{References}

1. Ambati, J. and B.J. Fowler, Mechanisms of age-related macular degeneration. Neuron, 2012. 75(1): p. 26-39.

2. Andreea Gheorghe, L.M.O.M., AGE-RELATED MACULAR DEGENERATION. Ophthalmology, 2015. 59(2): p. 74-7.

3. Al-Zamil, W. and S. Yassin, Recent developments in age-related macular degeneration: a review. Clinical interventions in aging, 2017. Volume 12: p. 1313-1330. 
4. Green, W.R., P.J. McDonnell and J.H. Yeo, Pathologic features of senile macular degeneration. Ophthalmology, 1985. 92(5): p. 615-27.

5. DeAngelis, M.M., et al., Genetics of age-related macular degeneration (AMD). Hum Mol Genet, 2017. 26(R1): p. R45-R50.

6. Layana, A.G., et al., Vitamin D and Age-Related Macular Degeneration. Nutrients, 2017. 9(10): p. 1120.

7. Handa, J.T., et al., A systems biology approach towards understanding and treating non-neovascular age-related macular degeneration. Nat Commun, 2019. 10(1): p. 3347.

8. Stahl, A., The Diagnosis and Treatment of Age-Related Macular Degeneration. Dtsch Arztebl Int, 2020. 117(29-30): p. 513-520.

9. Chandra, S., et al., Ten-year outcomes of antivascular endothelial growth factor therapy in neovascular age-related macular degeneration. Eye (Lond), 2020. 34(10): p. 1888-1896.

10. Cougnard-Gregoire, A., et al., Long-term blood pressure and age-related macular degeneration: the ALIENOR study. Invest Ophthalmol Vis Sci, 2013. 54(3): p. 1905-12.

11. Zuo, X., et al., Relationship between neural functional connectivity and memory performance in agerelated macular degeneration. Neurobiol Aging, 2020. 95: p. 176-185.

12. O'Connor, E.E. and T.A. Zeffiro, Why is Clinical fMRI in a Resting State? Front Neurol, 2019. 10: p. 420.

13. Chen, H.J., et al., Resting-state functional connectivity abnormalities correlate with psychometric hepatic encephalopathy score in cirrhosis. Eur J Radiol, 2015. 84(11): p. 2287-95.

14. Chen, J., et al., Effectiveness and neural mechanisms of home-based telerehabilitation in patients with stroke based on $\mathrm{fMRI}$ and DTI: A study protocol for a randomized controlled trial. Medicine (Baltimore), 2018. 97(3): p. e9605.

15. Prodoehl, J., R.G. Burciu and D.E. Vaillancourt, Resting state functional magnetic resonance imaging in Parkinson's disease. Curr Neurol Neurosci Rep, 2014. 14(6): p. 448.

16. Guo, Z., et al., Abnormal degree centrality in Alzheimer's disease patients with depression: A restingstate functional magnetic resonance imaging study. Exp Gerontol, 2016. 79: p. 61-6.

17. Li, Q.G., et al., Alterations of resting-state functional network centrality in patients with asthma: evidence from a voxel-wise degree centrality analysis. Neuroreport, 2018. 29(14): p. 1151-1156.

18. Hua, K., et al., Abnormal degree centrality in chronic users of codeine-containing cough syrups: A resting-state functional magnetic resonance imaging study. Neuroimage Clin, 2018. 19: p. 775-781.

19. Cai, F., et al., Network Centrality of Resting-State fMRI in Primary Angle-Closure Glaucoma Before and After Surgery. PLoS One, 2015. 10(10): p. e0141389.

20. Huang, X., et al., Disturbed spontaneous brain activity pattern in patients with primary angle-closure glaucoma using amplitude of low-frequency fluctuation: a fMRI study. Neuropsychiatr Dis Treat, 2015. 11: p. 1877-83.

21. Zhu, P.W., et al., Altered intrinsic functional connectivity of the primary visual cortex in youth patients with comitant exotropia: a resting state fMRI study. Int J Ophthalmol, 2018. 11(4): p. 668-673. 
22. Yan, X., et al., Altered Functional Connectivity of the Primary Visual Cortex in Adult Comitant Strabismus: A Resting-State Functional MRI Study. Curr Eye Res, 2019. 44(3): p. 316-323.

23. Chao-Gan, Y. and Z. Yu-Feng, DPARSF: A MATLAB Toolbox for "Pipeline" Data Analysis of RestingState fMRI. Front Syst Neurosci, 2010. 4: p. 13.

24. Zuo, X.N., et al., Network centrality in the human functional connectome. Cereb Cortex, 2012. 22(8): p. $1862-75$.

25. Zhang, B., et al., Altered spontaneous brain activity pattern in patients with ophthalmectomy: an resting-state fMRI study. Int J Ophthalmol, 2020. 13(2): p. 263-270.

26. Shao, Y., et al., Altered intrinsic regional spontaneous brain activity in patients with optic neuritis: a resting-state functional magnetic resonance imaging study. Neuropsychiatr Dis Treat, 2015. 11: p. 3065-73.

27. Tan, G., et al., A functional MRI study of altered spontaneous brain activity pattern in patients with congenital comitant strabismus using amplitude of low-frequency fluctuation. Neuropsychiatr Dis Treat, 2016. 12: p. 1243-50.

28. Huang, X., et al., Altered spontaneous brain activity pattern in patients with high myopia using amplitude of low-frequency fluctuation: a resting-state fMRI study. Neuropsychiatr Dis Treat, 2016. 12: p. 2949-2956.

29. Bogousslavsky, J., et al., Lingual and fusiform gyri in visual processing: a clinico-pathologic study of superior altitudinal hemianopia. J Neurol Neurosurg Psychiatry, 1987. 50(5): p. 607-14.

30. Kurcyus, K., et al., Opposite Dynamics of GABA and Glutamate Levels in the Occipital Cortex during Visual Processing. J Neurosci, 2018. 38(46): p. 9967-9976.

31. Sanda, N., et al., Visual brain plasticity induced by central and peripheral visual field loss. Brain Struct Funct, 2018. 223(7): p. 3473-3485.

32. Zeki, S., et al., A direct demonstration of functional specialization in human visual cortex. J Neurosci, 1991. 11(3): p. 641-9.

33. Zhang, B., et al., Altered spontaneous brain activity pattern in patients with ophthalmectomy: an resting-state fMRI study. Int J Ophthalmol, 2020. 13(2): p. 263-270.

34. Weiner, K.S. and K. Zilles, The anatomical and functional specialization of the fusiform gyrus. Neuropsychologia, 2016. 83: p. 48-62.

35. Hadjikhani, N., et al., Activation of the fusiform gyrus when individuals with autism spectrum disorder view faces. Neuroimage, 2004. 22(3): p. 1141-50.

36. Trimmel, K., et al., Left temporal lobe language network connectivity in temporal lobe epilepsy. Brain, 2018. 141(8): p. 2406-2418.

37. Onitsuka, T., et al., Fusiform gyrus volume reduction and facial recognition in chronic schizophrenia. Arch Gen Psychiatry, 2003. 60(4): p. 349-55.

38. Mangun, G.R., et al., ERP and fMRI measures of visual spatial selective attention. Hum Brain Mapp, 1998. 6(5-6): p. 383-9. 
39. Onitsuka, T., et al., Middle and inferior temporal gyrus gray matter volume abnormalities in chronic schizophrenia: an MRI study. Am J Psychiatry, 2004. 161(9): p. 1603-11.

40. Grotheer, M., B. Jeska and K. Grill-Spector, A preference for mathematical processing outweighs the selectivity for Arabic numbers in the inferior temporal gyrus. Neuroimage, 2018. 175: p. 188-200.

41. Schoenberg, M.R., et al., Neuropsychology Outcomes Following Trephine Epilepsy Surgery: The Inferior Temporal Gyrus Approach for Amygdalohippocampectomy in Medically Refractory Mesial Temporal Lobe Epilepsy. Neurosurgery, 2018. 82(6): p. 833-841.

42. Stroup, E., et al., Predicting verbal memory decline following anterior temporal lobectomy (ATL). Neurology, 2003. 60(8): p. 1266-73.

43. Chelune, G.J., Hippocampal adequacy versus functional reserve: predicting memory functions following temporal lobectomy. Arch Clin Neuropsychol, 1995. 10(5): p. 413-32.

44. Hermann, B.P., et al., Pathological status of the mesial temporal lobe predicts memory outcome from left anterior temporal lobectomy. Neurosurgery, 1992. 31(4): p. 652-6; discussion 656-7.

45. Dunlop, B.W., et al., Functional Connectivity of the Subcallosal Cingulate Cortex And Differential Outcomes to Treatment With Cognitive-Behavioral Therapy or Antidepressant Medication for Major Depressive Disorder. Am J Psychiatry, 2017. 174(6): p. 533-545.

46. Haznedar, M.M., et al., Cingulate gyrus volume and metabolism in the schizophrenia spectrum. Schizophr Res, 2004. 71(2-3): p. 249-62.

47. Jones, B.F., et al., Differential regional atrophy of the cingulate gyrus in Alzheimer disease: a volumetric MRI study. Cereb Cortex, 2006. 16(12): p. 1701-8.

48. Oliveri, M., et al., Parieto-frontal interactions in visual-object and visual-spatial working memory: evidence from transcranial magnetic stimulation. Cereb Cortex, 2001. 11(7): p. 606-18.

49. Dong, J.W., et al., fMRI activation in the middle frontal gyrus as an indicator of hemispheric dominance for language in brain tumor patients: a comparison with Broca's area. Neuroradiology, 2016. 58(5): p. 513-20.

50. Japee, S., et al., A role of right middle frontal gyrus in reorienting of attention: a case study. Front Syst Neurosci, 2015. 9: p. 23.

51. Gohel, S., et al., Resting-State Functional Connectivity of the Middle Frontal Gyrus Can Predict Language Lateralization in Patients with Brain Tumors. AJNR Am J Neuroradiol, 2019. 40(2): p. 319-325.

52. Mesulam, M.M. and E.J. Mufson, Insula of the old world monkey. III: Efferent cortical output and comments on function. J Comp Neurol, 1982. 212(1): p. 38-52.

53. Nagai, M., K. Kishi and S. Kato, Insular cortex and neuropsychiatric disorders: a review of recent literature. Eur Psychiatry, 2007. 22(6): p. 387-94.

54. Hu, Y.X., et al., Abnormal resting-state functional network centrality in patients with high myopia: evidence from a voxel-wise degree centrality analysis. Int J Ophthalmol, 2018. 11(11): p. 1814-1820. 
55. Gujing, L., et al., Increased Insular Connectivity and Enhanced Empathic Ability Associated with Dance/Music Training. Neural Plast, 2019. 2019: p. 9693109.

\section{Tables}

Due to technical limitations, Table 1 is only available as a download in the Supplemental Files section.

Table 2 Significant differences in DC between groups

\begin{tabular}{|c|c|c|c|c|c|c|c|}
\hline \multirow[t]{2}{*}{ Brain areas } & \multirow[t]{2}{*}{$\mathrm{L} / \mathrm{R}$} & \multirow[t]{2}{*}{ BA } & \multicolumn{3}{|c|}{$\begin{array}{l}\text { MNI } \\
\text { coordinates }\end{array}$} & \multirow[t]{2}{*}{ Voxels } & \multirow[t]{2}{*}{$t$} \\
\hline & & & $x$ & $\mathrm{Y}$ & Z & & \\
\hline \multicolumn{8}{|l|}{$\mathrm{HC} \bowtie \mathrm{AMD}$} \\
\hline Fusiform Gyrus & B & 37 & 39 & -24 & -36 & 408 & -4.636 \\
\hline Inferior Temporal Gyrus & B & 20 & -27 & -21 & -39 & 569 & -5.2586 \\
\hline Subcallosal Gyrus & B & 36 & -3 & -9 & -21 & 167 & -4.6032 \\
\hline Middle Frontal Gyrus & B & $10 \rrbracket 46$ & 12 & 39 & -30 & 163 & -4.7596 \\
\hline $\begin{array}{l}\text { Frontal_Mid_Orb_L (aal)/Insula/Inferior } \\
\text { Frontal Gyrus }\end{array}$ & L & $\begin{array}{l}12 \bigotimes 47 \rrbracket \\
13 \otimes 11\end{array}$ & 36 & -39 & 0 & 1729 & -4.5632 \\
\hline \multicolumn{8}{|l|}{$\mathrm{HC} \rrbracket \mathrm{AMD}$} \\
\hline Lingual Gyrus & B & 18 & 3 & -81 & -6 & 1545 & 5.0328 \\
\hline
\end{tabular}

Notes: The statistical threshold was set at voxel with $\mathrm{P}<0.05$ for multiple comparisons usingGaussian random field (GRF) theory ( $z>2.3, P<0.01$, cluster $>40$ voxels, AlphaSim corrected). Abbreviations: DC, Degree centrality; HC, Health controls; AMD, age-related macular degeneration; BA, Brodmann area; MNI, Montreal Neurological Institute; L, left; R, right; B, bilateral; Frontal_Mid_Orb, orbital middle frontal gyrus.

Table 3 Brain regions changes and its potential impact

Abbreviations: AMD, age-related macular degeneration; HCs, healthy controls; Frontal_Mid_Orb_L, left orbital middle frontal gyrus.

\section{Figures}




\begin{tabular}{|c|c|c|c|}
\hline Brain regions & $\begin{array}{l}\text { Experimental } \\
\text { result }\end{array}$ & Brain function & $\begin{array}{l}\text { Anticipated } \\
\text { results }\end{array}$ \\
\hline Lingual gyrus & AMDs $₫ \mathrm{HCs}$ & Visual processing & $\begin{array}{l}\text { Visual } \\
\text { impairment }\end{array}$ \\
\hline Fusiform gyrus & AMDs $₫ \mathrm{HCs}$ & $\begin{array}{l}\text { Face recognition } \llbracket \text { object secondary } \\
\text { classification recognitio }\end{array}$ & $\begin{array}{l}\text { Visual } \\
\text { impairment } \\
\text { and mental } \\
\text { illness }\end{array}$ \\
\hline Inferior temporal gyrus & AMDs $\bowtie H C s$ & $\begin{array}{l}\text { Language and semantic memory } \\
\text { processing, visual } \\
\text { perception,multimodal sensory } \\
\text { integration }\end{array}$ & $\begin{array}{l}\text { Memory } \\
\text { impairment }\end{array}$ \\
\hline Subcallosal gyrus & AMDs $₫ \mathrm{HCs}$ & Part of the default model network & $\begin{array}{l}\text { Depression } \\
\text { and anxiety }\end{array}$ \\
\hline Middle frontal gyrus & AMDs『HCs & $\begin{array}{l}\text { Memory processing, } \\
\text { attention control }\end{array}$ & $\begin{array}{l}\text { Visual } \\
\text { impairment }\end{array}$ \\
\hline $\begin{array}{l}\text { Frontal_Mid_Orb_L } \\
\text { (aal)/Insula/Inferior } \\
\text { Frontal Gyrus }\end{array}$ & AMDs $邓 \mathrm{HCs}$ & $\begin{array}{l}\text { Part of the default model } \\
\text { network,converge information,eye } \\
\text { movement, visual attention }\end{array}$ & $\begin{array}{l}\text { Mental } \\
\text { disorders }\end{array}$ \\
\hline
\end{tabular}



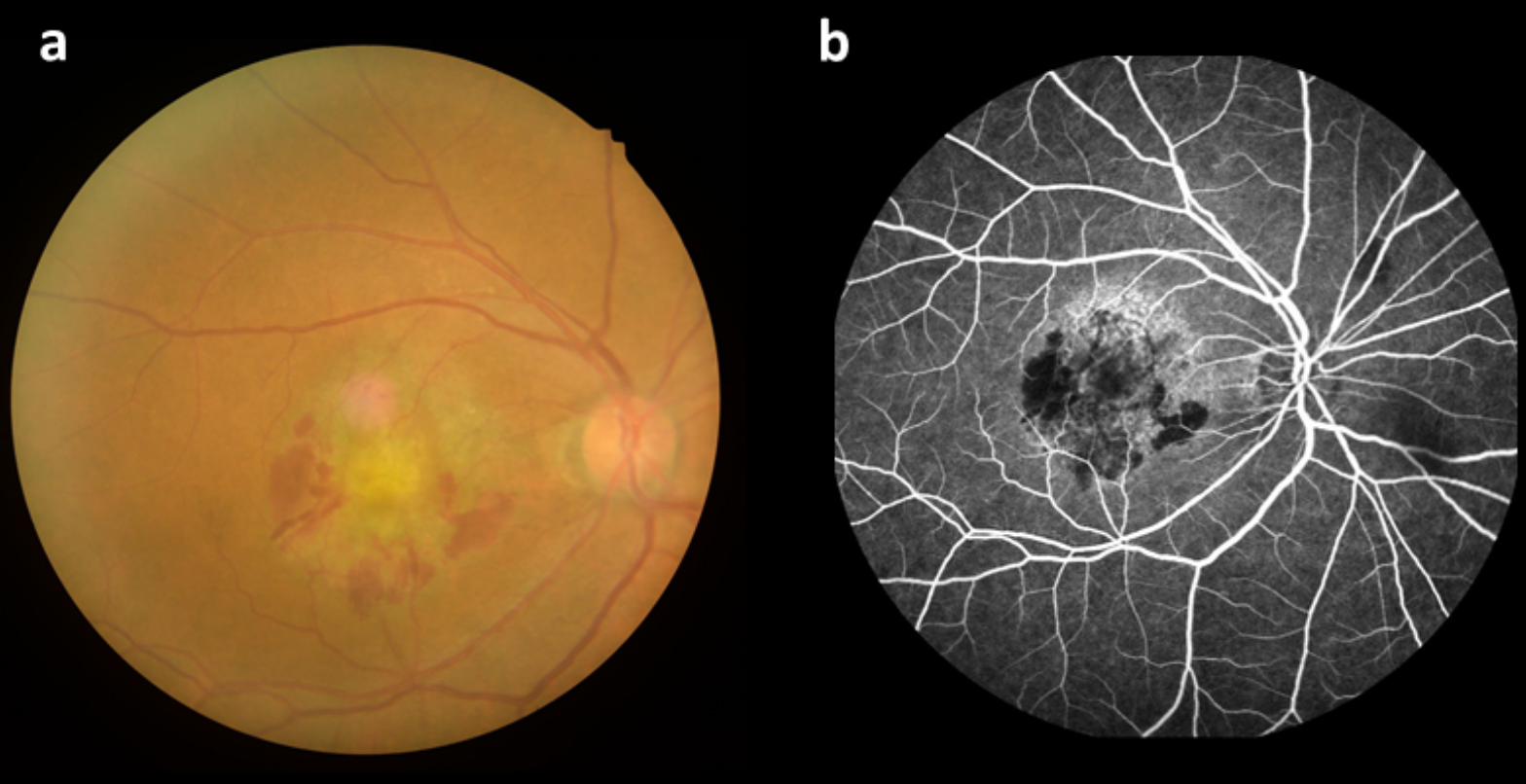

\section{Figure 1}

Funduscopy (a) showed that there were yellowish-white lesions in the macular area with more hard exudates around. The fluorescein fundus angiography (b) showed mottled high fluorescence in the macular area. 


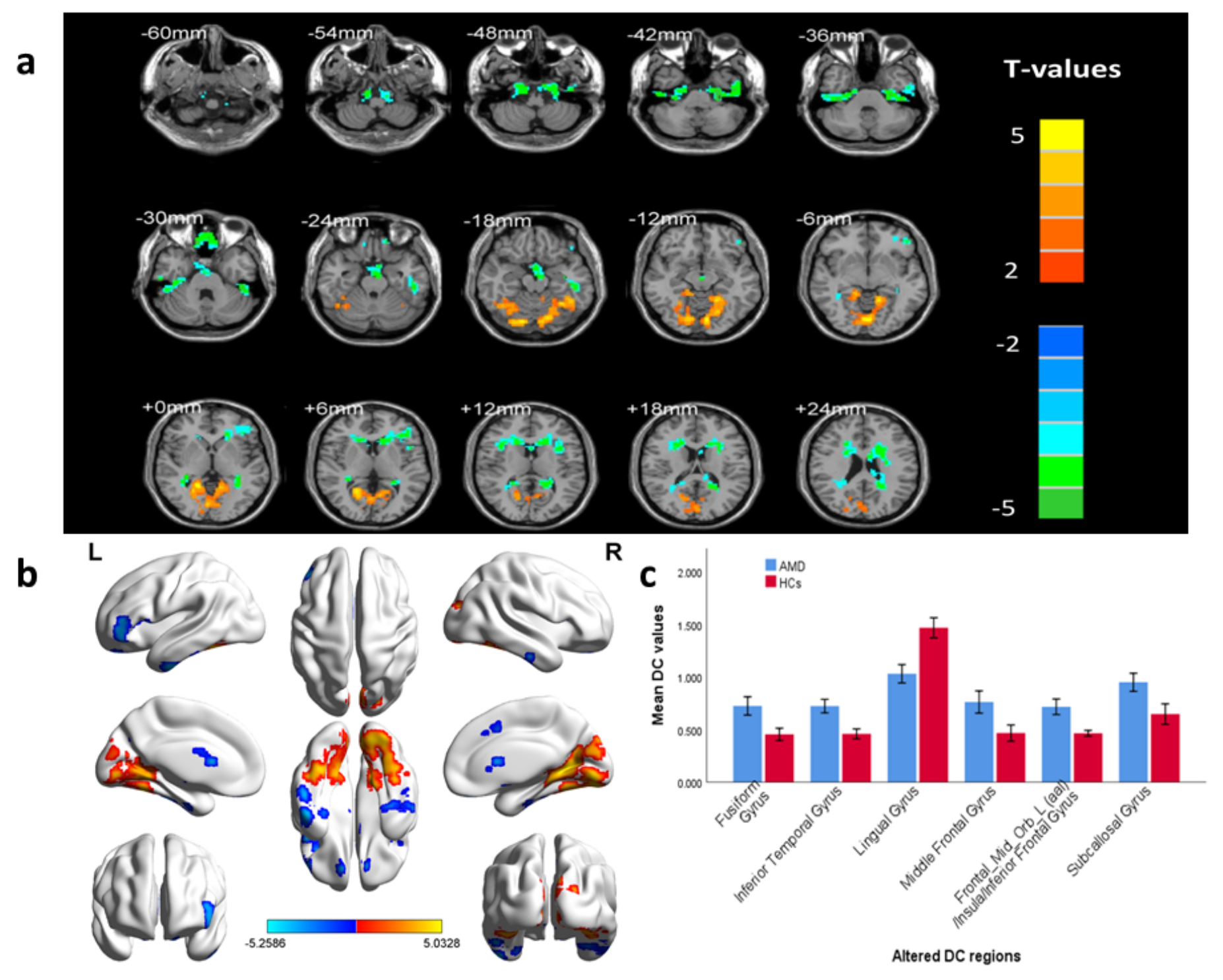

Figure 2

Spontaneous brain activities of AMD and healthy controls We observed the bilateral fusiform gyrus, bilateral middle frontal gyrus, bilateral subcallosal gyrus,bilateral inferior temporal gyrus, bilateral lingual gyrus and left orbital middle frontal gyrus/insular gyrus/inferior frontal gyrus. Brain areas with higher DC values are represented by yellow or red, while lower DC values are represented by green or blue. $\mathrm{XP}<0.001$, cluster $>40$ voxels, AlphaSim corrected》 

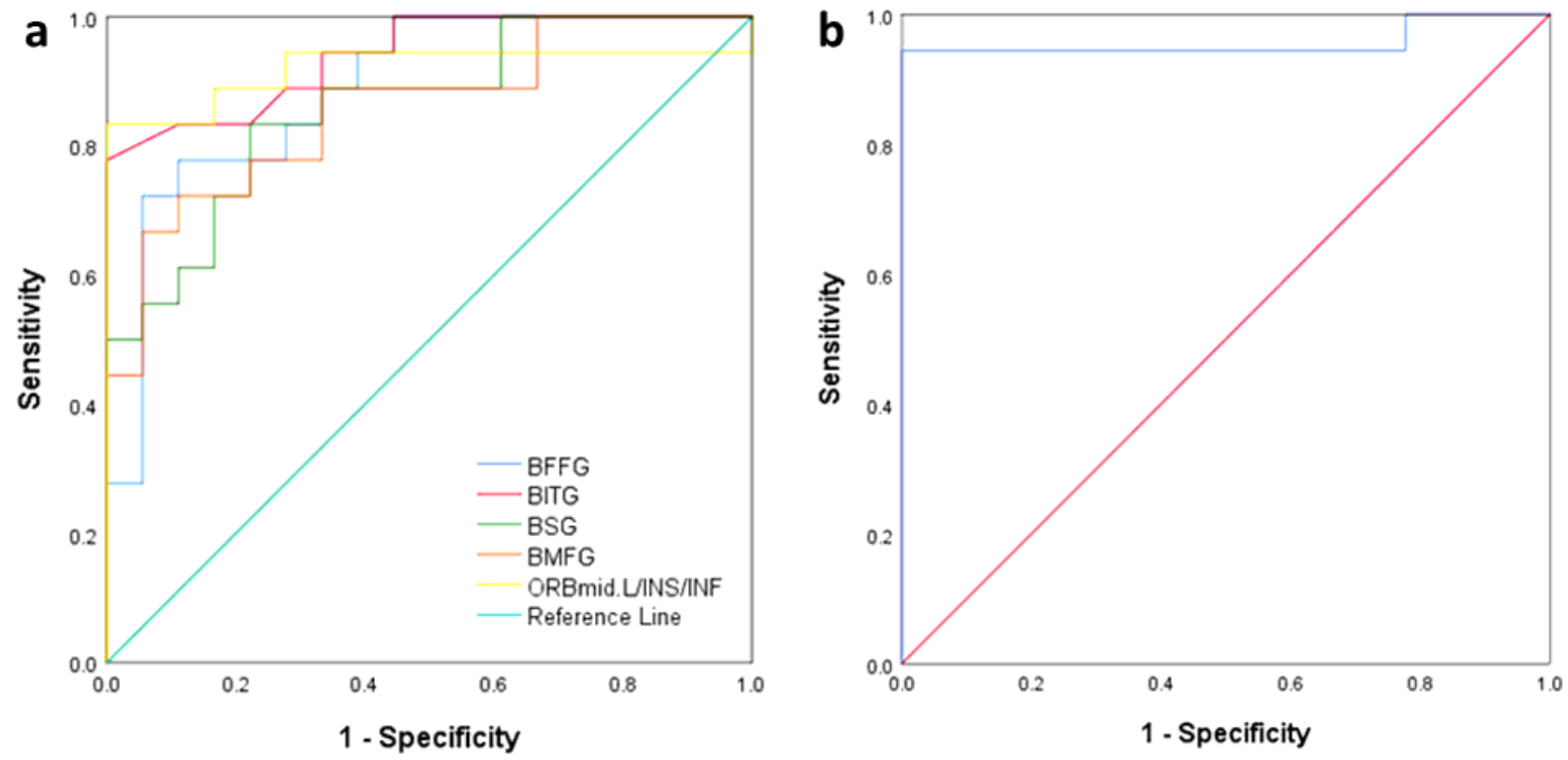

Figure 3

ROC curve analysis of the mean DC values for altered brain regions The AUCs for DC values: BFFG: 0.889, BITG: 0.940, BSG: 0.861, BMFG: 0.858, ORBmid.L/INS/ORB: 0.920, BLING: 0.957.BFFG: bilateral fusiform Gyrus;BITG: bilateral inferior temporal gyrus; BMFG: bilateral middle frontal gyrus; BSG: bilateral subcallosal gyrus; ORBmid.L/INS/INF: middle frontal gyrus,orbital.left/insular gyrus/inferior frontal gyrus;BLIFG: bilateral lingual gyrus. 


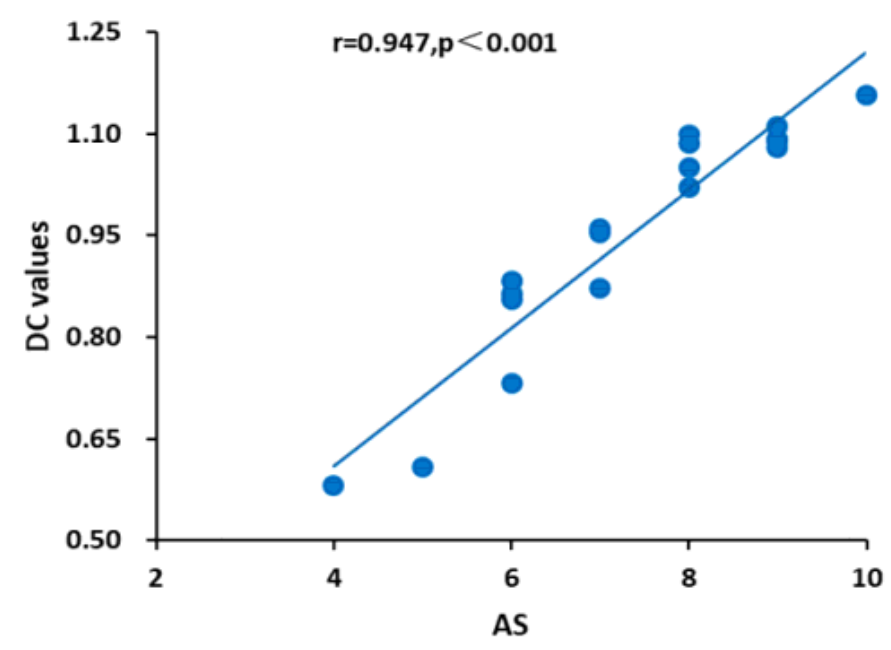

(a)

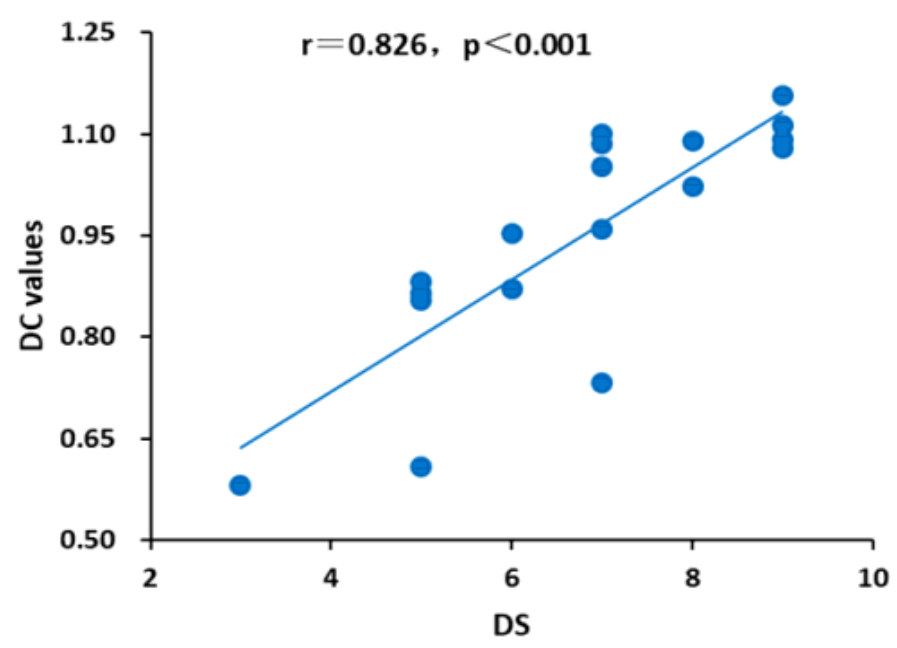

(b)

\section{Figure 4}

Correlations between the average DC values of the subcallosal gyrus and the clinical characteristics. Both

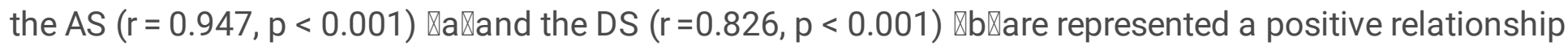
with the $D C$ values of the subcallosal gyrus. 


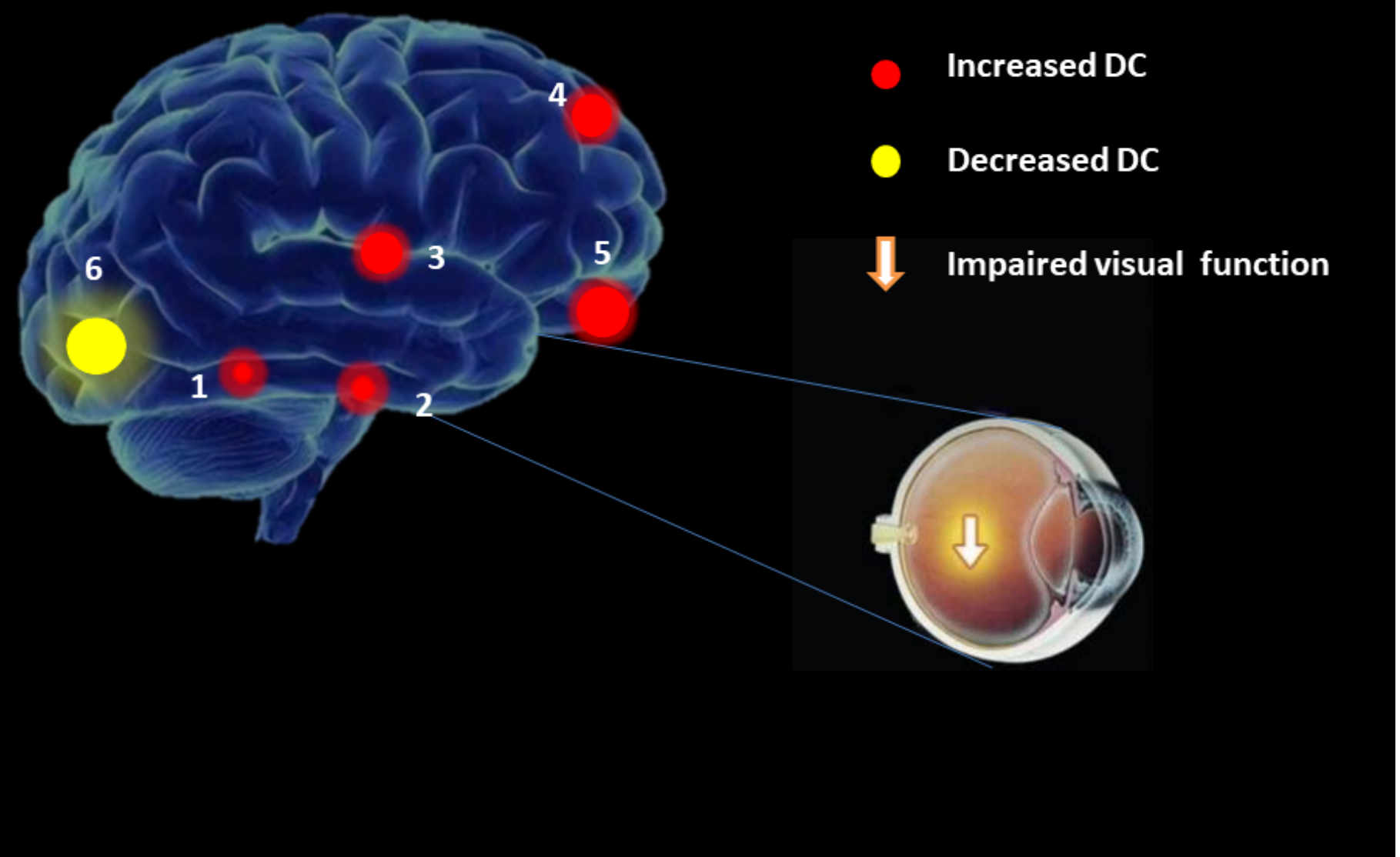

\section{Figure 5}

DC results of brain activity in AMD group The DC values of the cerebrum areas in AMD group were in the following: 1- bilateral fusiform gyrus ( $t=-4.636), 2$ - bilateral inferior temporal gyrus ( $t=-5.2586), 3$ - bilateral subcallosal gyrus ( $t=-4.6032)$, 4- left middle frontal gyrus ( $t=-4.7396), 5$ - left orbital middle frontal gyrus/insular gyrus/inferior frontal gyrus ( $t=-4.5632)$, 6- bilateral lingual gyrus $(t=5.0328)$. The intensity of brain activity is reflected by the size of spots. 


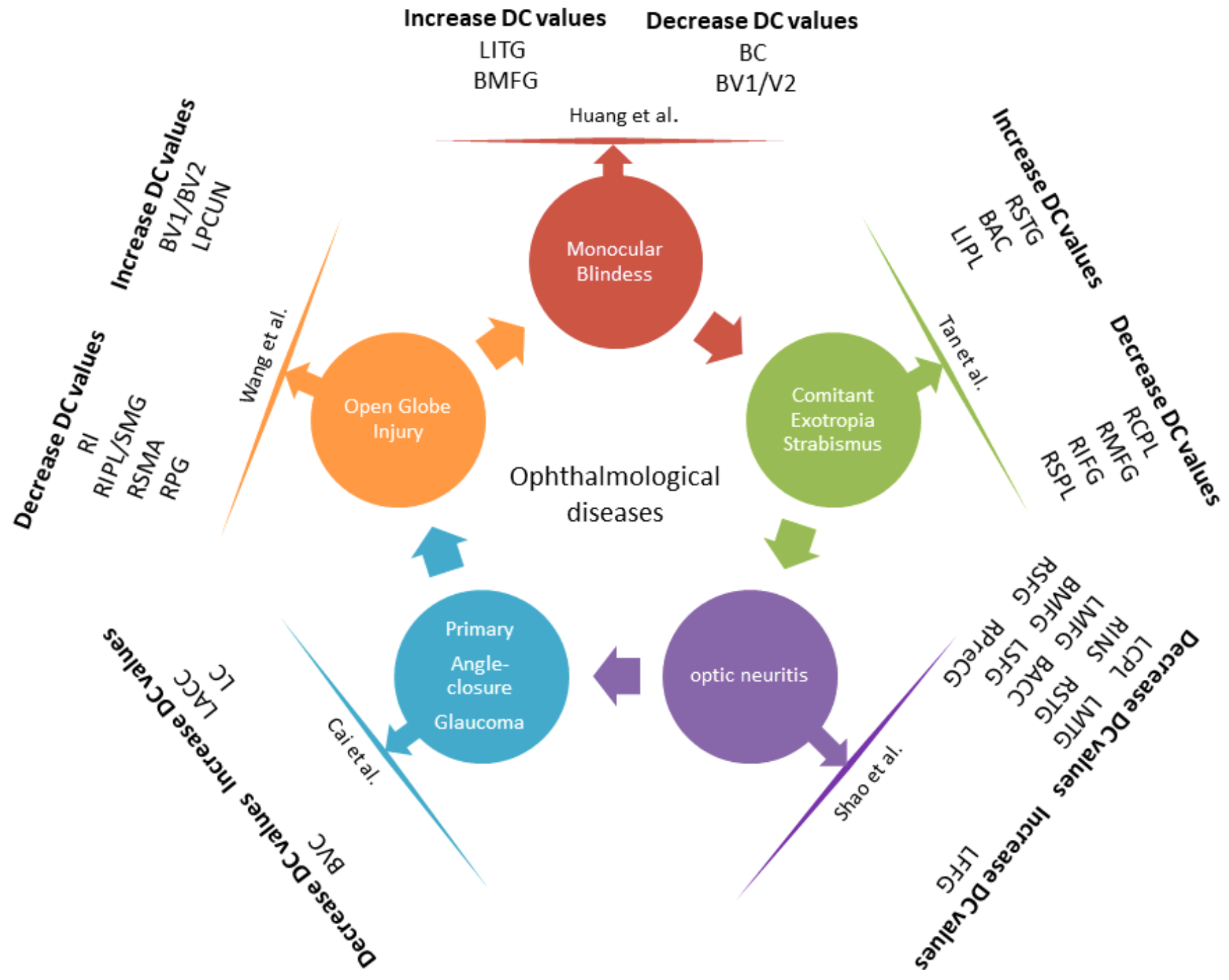

Figure 6

DC method applied in ophthalmological diseases.

\section{Supplementary Files}

This is a list of supplementary files associated with this preprint. Click to download.

- Table1.doc 